\title{
The Effects of Social Interaction and Social Norm Compliance in Pay-What-You-Want Situations
}

\author{
Leon Paul Hilbert, Augustin Suessmair \\ Leuphana University of Lueneburg, Lueneburg, Germany \\ Email: leon.hilbert@stud.leuphana.de, suessmair@uni.leuphana.de
}

Received 15 July 2015; accepted 18 August 2015; published 21 August 2015

Copyright (C) 2015 by authors and Scientific Research Publishing Inc.

This work is licensed under the Creative Commons Attribution International License (CC BY). http://creativecommons.org/licenses/by/4.0/

c) (i) Open Access

\begin{abstract}
Since Pay-What-You-Want is a relatively new field of study, the influence factors during the pricing process are not sufficiently explored. It was hypothesized that in a hypothetical Pay-What-YouWant situation, increased social interaction and social norm compliance would lead to a higher willingness to pay for a travel mug. In a laboratory experiment, 79 German students were randomly assigned to three groups which varied in the degree of social interaction. Social norm compliance was assessed with a questionnaire. A $3 \times 3$ between-group factorial ANOVA showed a significant main effect for social interaction $\left(p=0.025, \eta^{2}=0.08\right)$, whilst social norm compliance was slightly not significant $\left(p=0.067, \eta^{2}=0.03\right)$. Follow-up comparisons were calculated and the results discussed. The findings implicate that especially the degree of social interaction should be regarded, both by researchers and practitioners, as an important situational factor influencing the price in a Pay-What-You-Want situation.
\end{abstract}

\section{Keywords}

Pay-What-You-Want, Social Interaction, Social Norms, Willingness to Pay

\section{Introduction}

Finding the right price for goods and services is a major challenge for sellers in all businesses. The participative pricing mechanism Pay-What-You-Want (PWYW) seems to be an unconventional alternative to address that challenge. Instead of focusing on the seller, it sets the buyer in full control over the pricing process, empowering him to set the price for the offered product completely on his own [1]. Additionally, the seller is not able to reject a buyer's offer, even though the range of possible prices includes zero [1]. 
Following the concept of the homo oeconomicus, one might expect the buyer to selfishly pay a prize of zero. Contrary to this, numerous real world applications have turned out to be profitable for the seller. The best known example for a successful PWYW campaign is the release of the Radiohead album In Rainbows in 2007. The band published the album on its own website and let the customers set the buying price. They sold up to three million records, earning more money than with all prior albums combined [2]. Other artists followed their campaign successfully [3]. As an example for the service sector, the IBIS Hotel in Singapore successfully used PWYW pricing as an opening campaign [4]. In December 2012, a zoo in Muenster, Germany, changed its pricing mechanism to PWYW. Over 56,000 visitors made use of the offer, increasing the zoo's revenues by 2.5 times compared to December 2011 [5].

All these examples have in common that they were short time offers only. Hence, they could have been positively affected by pioneer effects that do not last over time [6]. However, the online platform Humble Bundle has successfully employed PWYW pricing mechanisms for years. Since 2010, they have sold packages of digital content for more than 110 million dollars [7]. Moreover, Riener and Traxler [8] were the first to conduct longterm studies of PWYW effects, monitoring the revenues in an Austrian restaurant. Over two years, the average payment decreased slightly, while the number of daily guests increased, leading to positive revenues overall. Until today, there is clear evidence that Pay-What-You-Want pricing can be a profitable alternative pricing mechanism [9]-[12].

The first researchers to investigate the seemingly surprising success of PWYW applications were Kim et al. [1]. Besides giving a definition and classification of PWYW pricing, they examined which factors influenced the price in PWYW situations. They conducted three field experiments in the service sector (restaurant, cinema, delicatessen), whereby the total revenues under PWYW pricing increased in two cases. Furthermore Kim et al. [1] found seven factors influencing the price that people paid. In all three experiments, the customers' reference price, income, price consciousness, satisfaction, and fairness motives seemed to be main drivers in the pricing process. Moreover, altruism and loyalty were found to be important contributors in one of the three studies. Henceforward, those seven factors were mainly accepted and assumed for further research [13] [14].

Nevertheless, Kunter [12] correctly stated that the seven factors by Kim et al. [1] where not derived from a conceptual framework and are therefore not necessarily exhaustive. For instance, Kim et al. [1] did not regard compliance to social norms as an independent factor on the price although they used it as main explanation for possible positive revenues in PWYW situations. In that regard, Regner [15] found that social norms play an important role in PWYW pricing in addition to fairness motives. In his field experiment, participants' expectations about what others thought about their payments influenced the pricing process. Likewise, Jung, Nelson, A. Gneezy and Gneezy [16] found that participants relied heavily on the real or perceived behaviour of others during PWYW pricing. Furthermore, an experiment by Mak, Zwick, Rao and Pattaratanakun [17] revealed that over several rounds, social pressure from other buyers led to increased payments from those paying a below average price. Over the course of five studies, findings by Kunter [12] showed that the avoidance of feelings of guilt was a pivotal factor during PWYW pricing. According to the author, guilt was triggered through a self-observed violation of social norms (e.g. paying to little). Feelings of shame which occurred when a violation of social norms was seemingly observed by others were also found to be relevant factors in some of Kunter's [12] studies. Moreover, Dorn and Suessmair [18] found that social agreeableness had an effect on the willingness to pay in an online PWYW experiment. Social agreeableness was measured as the expectation of what others think about oneself when deciding how much to pay.

Altogether, the recent research indicates that compliance to social norms seems to be an important additional factor in PWYW pricing that goes beyond fairness motives, consumer loyalty and altruism. This assumption is additionally supported by findings concerning the effect of social norms compliance in related fields such as tipping behaviour [19] or social dilemmas [20].

It can further be assumed that social interaction has an influence in PWYW pricing. Again, results from related economic fields such as dictator games [21]-[23], public goods experiments [24] [25] and donations [26] [27] show clear evidence for increasing payments under higher degrees of social interaction. However, the effect of social interaction on the price in PWYW situations has seldom been studied and the results obtained so far are inconclusive. For example, Regner and Riener [6] conducted a field experiment on buyer-seller interactions in an online music store. They found that a reduction of anonymity between the buyer and the seller led to an increase of mean payments but a reduced number of overall purchases, resulting in a loss of revenues. On the other hand, an online experiment conducted by Dorn and Suessmair [18] revealed that increased imagined 
buyer-seller interaction led to a higher willingness to pay for a McDonald's BigMac in a hypothetical setting.

Kim et al. [11] also tested the effect of buyer-seller interactions on PWYW pricing in an online experiment and two field studies. In the experiment, the degree of social interaction was varied from an anonymous online purchase to a personal buyer-seller relationship. While the effect was found to be highly significant in the online experiment, the field studies in a French restaurant and a cafeteria showed no significant effects of social interaction on the price paid. The authors explain these findings with the problem of creating an anonymous setting in a restaurant or cafeteria where personal interaction between the waiter and the guests can hardly be avoided.

In an analogue field experiment, A. Gneezy et al. [3] tried to avoid this problem by letting the customers in the anonymous condition pay by putting money in an envelope. The customers in the "observed" setting paid at the counter. Surprisingly, the results revealed that payments in the anonymous condition were slightly but significantly higher. This could be explained by the fact that customers in the anonymous condition received $€ 20$ in change that they could use to pay exactly the price they wanted. Hence, it can be argued that anchor effects have interfered in the pricing process [28].

In conclusion, the literature shows mixed evidence on the effects of social interactions on PWYW pricing. Each hypothetical online experiment and field study faced problems in controlling social interaction as an independent variable. To address this problem, the present study aims to examine the effects of social interaction in a controlled laboratory PWYW experiment. Moreover, it aims to further examine the effects that social norm compliance has on the price in PWYW situations. Therefore, a laboratory experiment is conducted in which participants can set their own price for a travel mug. The degree of social interaction is varied over three steps, namely from anonymous pricing over a setting where the price has to be named to the experimenter to a setting where the price has to be named to the experimenter in the presence of a second participant. Furthermore, the influence of social norm compliance in each situation is assessed through a questionnaire. It is hypothesized that higher degrees of social interaction lead to a higher willingness to pay in the PWYW situation. It is furthermore hypothesized that social norm compliance has a positive effect on the willingness to pay.

\section{Method}

\subsection{Participants}

The participants (60 women, 19 men, $M_{\text {age }}=21.52$ years, $S D_{\text {age }}=3.12$ years, range: 19 - 38 years) were recruited through course newsletters and lectures at the Leuphana University in Germany. All participants were enrolled students, all but two studying Business Psychology. The mean net income of the participants including student loans and child benefits was $€ 634.55$ ( $S D=232.39$ ). The participants received a certificate for their attendance which is mandatory for Business Psychology students.

\subsection{Design}

To address the research hypotheses, a 3 (Social Interaction: low, medium, high) $\times 3$ (Social Norm Compliance: low, medium, high) between-group factorial ANOVA on the willingness to pay for a travel mug in a PWYW Situation was used.

Social Interaction was a between-participant variable and reflected how much the participant interacted with other people during the pricing process. In the low-interaction group, participants did not interact with anyone during the whole pricing process. In the medium group, the participants interacted with the experimenter while setting the price. In the high condition, two participants were present at the same time. They had to interact with the experimenter in the presence of the other participant.

To analyse the second factor, participants' social norm compliance was assessed using a questionnaire. To transform the continuous measure into a categorical variable, participants were later divided in three percentile groups.

\subsection{Materials}

Whilst the majority of PWYW research has been done on either digital goods [10] [14] [15] or in the service sector [1] [3] [8], a travel mug was chosen for the present experiment. It was assumed that all participants would be familiar with travel mugs which was confirmed in the pre-test. The travel mug was manufactured by the German company ISOSTEEL. In 2007, the recommended retail price for the travel mug was €17.95. Hence, the 
chosen product ranks in the upper middle-class for travel mugs.

\subsection{Measures}

The willingness to pay for the travel mug constituted the dependent variable. Findings by Marett et al. [14] indicated that willingness to pay overestimates the price that would actually be paid in a PWYW situation. Therefore, the willingness to pay in the present experiment was not used to gain insights into total revenues for the travelling mug compared to alternative pricing mechanisms. However, the interpretability of group differences is not affected by this limitation and has been used in other studies [13]. Social Norm Compliance was measured on a scale consisting of three items, (Table 1) on five-point Likert scales, ranging from " 1 : totally disagree" to "5: totally agree". In the pre-test, the scale had a good internal consistency (Cronbach's $\alpha=0.75$ ). The average of the three items constituted the score for each participant and was later used to allocate them to three percentile groups, leading to a low (SNC 1: $\leq 2.33$ ), medium (SNC 2: $2.34-3.33$ ) and high group (SNC 3: $\geq 3.34$ ). The items were mixed with six other subscales measuring factors relevant in PWYW-Situations to distract participants from the research question. The factors altruism, fairness, income, price consciousness, satisfaction and reference price were adapted from Kim et al. [1] and measured in the same manner with three items each.

Even though these factors were proposed a few years ago and are widely accepted, there are still no validated scales to measure them. To address that challenge, the items were based on previous research studies [1] [14] and additionally tested in the pre-test. Thereby, the items were analysed for floor and ceiling effects and Cronbach's $\alpha$ was obtained to test the internal consistency for each scale (Table 1). As a result of the pre-test, the factor loyalty which was also introduced by Kim et al. [1] was dismissed and several items were reformulated.

In addition, the questionnaire contained explorative questions on the relevance of social norms. The participants were asked if they had considered any social norms during the pricing process and if so, to describe them and give examples. Furthermore, participants were asked if they experienced the PWYW-Situation to be pleasant and if they would prefer a non-participative pricing method. The participants were further asked to guess the retail price of the travel mug and to answer questions about their habits regarding the consumption of hot beverages. Those questions were posed to explain possible statistical spikes and to collect data for possible explorative research. The questionnaire finished with socio-demographic questions.

\subsection{Procedure}

The participants registered through a poll created with an online scheduling software. The poll was limited to a maximum of two persons for each time slot. The only information given beforehand was that the experiment was about a travel mug and would last for around 20 minutes. Those participants who enlisted solely were randomly allocated to the low and medium social interaction group whereas the participants who registered for the same time slot formed the high group. An anonymous registering hindered participants to arrange their pairing intentionally.

The experiment took place in a seminar room at the university. All participants were welcomed and thanked for their attendance. Afterwards they were asked to take their seat which was located sideways to the experimenter. The instruction, the questionnaire and the travel mug were already placed at the table. The participants were asked to read through the instruction in which the PWYW mechanism was described shortly. It was stated that the seller would like to use the travel mug to test a new pricing method in which the buyer was empowered to choose the price on his own. It was also stated that naming any price-including a price of $€ 0.00$ — would directly lead to a purchase. Participants received a product description that was provided by the manufacturer and were actively allowed to take closer inspections on the travel mug during the pricing process.

Participants in the low interaction group received the entire instruction in a written form and chose the price on their own, simulating an anonymous purchase. In the group with medium social interaction the part of the instruction concerning the pricing mechanism was read out by the experimenter and the participants were asked to name the price they were willing to pay. Thus, this group represented a buyer-seller situation. Participants in the high interaction condition attended the experiment pairwise. Again, the part of the instruction concerning the pricing mechanism was read out by the experimenter. This time both participants were told to choose their price on their own and note it on a blank paper. Beforehand, they were also informed that they would be asked to name their price to the experimenter when both participants had chosen their price. In that way, it was ensured that both participants were conscious about the interaction with the experimenter in the presence of the other 
Table 1. Scales and items for influence factors on the price in PWYW situations.

Scales and items (Cronbach’s $\alpha$ )

Social norm compliance $(\alpha=0.75)$

I did not want to pay less than respectable.

I thought about what others expected me to do.

I did not want to get negative attention by paying an inadequate price

Altruism $(\alpha=0.85)$

I wanted to be good towards the seller.

I wanted to help the seller.

I did not want to put my interests above those of the seller.

Fairness $(\alpha=0.93)$

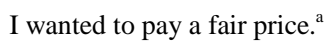

I did not want to be unfair towards the seller.

I wanted to pay a suitable price.

For me, fairness played no special part in the pricing process. ${ }^{\mathrm{b}}$

Income $(\alpha=0.96)$

I referred to my income.

I thought about how much money I have available.

I referred to the money I possess. ${ }^{\text {a }}$

I did not consider the amount of money I possess at all. ${ }^{\mathrm{b}}$

Price consciousness $(\alpha=0.79)$

I wanted to make a bargain buy.

I wanted to seize the opportunity to pay a minimized price for the product.

I did not want to spend to much money. ${ }^{\text {a }}$

Getting a cheap price was of secondary importance for me. ${ }^{\text {b }}$

Reference price $(\alpha=0.88)$

I imagined the average price of the product.

I had in mind how much I would have to pay in a regular store.

I thought about how much the product usually costs.

Satisfaction $(\alpha=0.72)$

I referred to the quality of the product.

I chose the price on the basis of my satisfaction with the product. ${ }^{\text {a }}$

I referred to my confidence in the product.

I thought about whether the product seemed high-class to me. ${ }^{\text {b }}$

Loyalty $^{*}(\alpha=-0.13)$

I would have paid a different price if I had been a regular customer.

I considered that this was a non-recurring offer.

I looked after my loyalty towards the seller.

$2.15 \quad 1.37$

Note: All results were taken from the pre-test. The items were translated from German. ${ }^{\mathrm{a}}$ Item was dropped after the pre-test. ${ }^{\mathrm{b}}$ Item was added after the pre-test. "The loyalty scale was dropped after the pre-test due to a lack of internal consistency. 
participant but not able to adjust their price to the other's price. Altogether, the last group simulated a buyerseller setting under observance of a third party.

After the pricing situation, participants were asked to complete the questionnaire described above. Subsequent to finishing the questionnaire, participants were repeatedly thanked for their attendance, received their certificate and were offered some sweets.

\section{Results}

All requirements to perform an ANOVA were met (Table 2 \& Table 3).

Results of the ANOVA on the willingness to pay for a travel mug in a Pay-What-You-Want situation revealed a significant main effect for social interaction, averaged over social norm compliance, $F(4,70)=3.87, p=0.025$, $\eta^{2}=0.08$. This was followed up by three pairwise main effect comparisons, indicating that the willingness to pay in the high social interaction group $(M=12.80, S D=5.01)$ was significantly higher than the willingness to pay in the low social interaction group $(M=9.15, S D=4.26), p=0.008$ (Figure 1). Moreover, the willingness to pay in the medium social interaction group $(M=11.92, S D=5.55)$ was significantly higher than the willingness to pay in the low social interaction group, $p=0.042$. There was no significant difference between the high and the medium social interaction group, $p=0.519$.

The main effect of social norm compliance on the price paid in the PWYW situation was slightly not significant, $F(4,70)=2.80, p=0.067, \eta^{2}=0.03$, indicating that people experiencing a low social norm compliance $(M$ $=9.58, S D=5.53)$ were willing to pay the same price as people experiencing a medium $(M=11.32, S D=5.06)$ or a high $(M=12.71, S D=4.63)$ social norm compliance.

There was no significant interaction between the social interaction and social norm compliance, $F(4,70)=$ $0.89, p=0.473, \eta^{2}=0.04$.

\section{Conclusions}

The aim of this study was to investigate the effects of social interaction and social norm compliance on the price in PWYW situations. Therefore, a buying situation for a travel mug was simulated under PWYW conditions in a laboratory experiment. It was hypothesized that an increased degree of social interaction in the pricing situation would lead to a higher willingness to pay. This hypothesis was confirmed because participants in the anonymous

Table 2. Kolmogorov-Smirnov-Test on normal distribution of the willingness to pay for each condition.

\begin{tabular}{cccc}
\hline Condition & $d f$ & Statistic & Sig $^{\mathrm{a}}$ \\
\hline Social interaction & & & \\
Low & 26 & 0.16 & 0.068 \\
Medium & 25 & 0.11 & $0.200^{\mathrm{b}}$ \\
High & 25 & 0.13 & $0.200^{\mathrm{b}}$ \\
Social norm compliance & & & \\
Low & 24 & 0.11 & $0.200^{\mathrm{b}}$ \\
Medium & 25 & 0.14 & 0.192 \\
High & 27 & 0.12 & $0.200^{\mathrm{b}}$ \\
\hline
\end{tabular}

Note: $d f$ = degrees of freedom. A normal distribution of the willingness to pay can be assumed in all conditions. ${ }^{\mathrm{a}}$ Lilliefors Significance Correction is used. ${ }^{\mathrm{b}}$ This is the lower bound of the true significance.

Table 3. Levene's test for equality of variances ${ }^{\mathrm{a}}$.

\begin{tabular}{ccccc}
\hline Dependent variable & $F$ & $d f 1$ & $d f 2$ & Sig. \\
\hline Willingness to pay & 0.43 & 8 & 70 & 0.901 \\
\hline
\end{tabular}

Note: $d f$ = degrees of freedom. Equality of variances can be assumed. ${ }^{\mathrm{a}}$ Design: Constant term + social interaction + social norm compliance + social interaction*social norm compliance. 


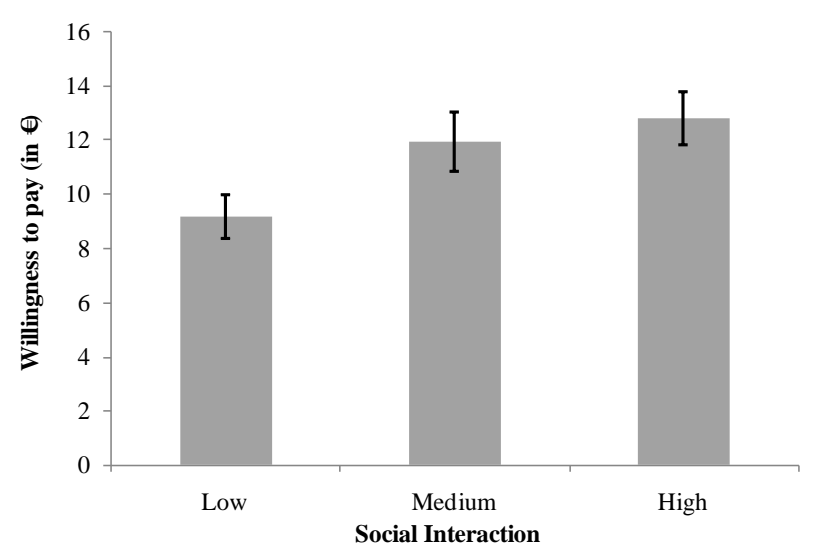

Figure 1. Means of the willingness to pay for different degrees of social interactions. Error bars represent standard error.

condition paid approximately $25 \%$ less than participants in the setting with buyer-seller interaction and those in the setting in which the buyer-seller interaction was additionally observed by a third party. This result supports findings from prior online experiments that indicated higher payments under increased social interaction [11] [18].

However, the results did not reveal a significant difference between the medium social interaction group in which the participant had to name the price to the experimenter and the high interaction group where an observing participant was added to the setting. These results are in contrast to findings by Dorn and Suessmair [18] who found that an additional third party observance increased payments compared to an unobserved buyer-seller interaction. As argued by Chang, Lusk and Norwood [29], hypothetical settings such as the ones used by Dorn and Suessmair [18] might have a weaker external validity than experimental settings. Hence, this difference in findings might be explained by the fact that the imagined impact of increasing social interaction could have been overestimated by the participants in the online experiment. However, both studies provide strong evidence on the general effect of social interaction in PWYW situations which need to be researched in further detail. For instance, it could be examined if different amounts and kinds of observers (e.g. unknown persons, friends, other buyers) vary in their impact on the price. Furthermore, the evidence for the effect of social interaction on the price in field experiments is mixed [3] [11] [15]. Hence, further research should be conducted to replicate the findings of this laboratory experiment in the field.

The second hypothesis stated that participants who comply stronger with social norms would state a higher willingness to pay. This hypothesis had to be rejected since no significant differences on the willingness to pay were found between participants who exhibited low, medium and high degrees of social norm compliance, contradicting findings from Jung et al. [16], Kunter [12] and Mak et al. [17]. Hence, the findings of this study indicate that social norm compliance is not a relevant factor in PWYW pricing. However, this interpretation has to be considered with caution. First of all, significance for the effect of social norm compliance was missed just slightly and the means show a pattern that follows the hypothesis. Participants with a low score on social norm compliance were willing to pay an average of €9.58, whilst those with medium scores averaged at $€ 11.32$ and those complying strongly with social norms at $€ 12.71$. Furthermore, social norm compliance was measured through self-assessment after the purchase which can be biased in general as shown by Dunning, Heath and Suls [30]. Precisely, since social norms can often be activated unconsciously [20], they could have affected the participants stronger than they have noticed. Therefore, further investigations on the influence of social norms could try to go beyond self-assessment. For example, one could try to actively manipulate social norms during the experiment. Following findings by Kunter [12], this could be done through explicit priming before the experiment, establishing feelings of guilt or shame through social norms.

Additionally, this study provides explorative indications of the profitability of PWYW pricing on consumer goods. Whilst theoretical illustrations by Kim et al. [1] give an overview on product and service qualities that could enhance profitability under PWYW pricing, the empirical validation for consumer goods is rather scarce. Disregarding the fact that the willingness to pay overestimates the actual price paid in a PWYW situation [14], the findings from this study imply that selling low to medium priced consumer goods under PWYW conditions 
could lead to positive revenues. In the present study, only two participants chose a price of $€ 0.00$ which was balanced out by more than $10 \%$ of the participants who were willing to pay more than the recommended retail price of $€ 17.95$ and two participants who chose to pay $€ 25$. To evaluate this implication, field studies should try to investigate the total revenues of consumer goods under PWYW pricing.

In conclusion, this study presents important findings for both researchers and practitioners. Given that this is the first study to manipulate social interaction in a laboratory experiment, the findings help to understand its effect in PWYW situations. The results implicate that the degree of social interaction is as an important influence factor in PWYW pricing and should be added to the prevalent seven factors established by Kim et al. (2009). Thus, the integration of social interaction would add a situational factor to the established person-related (e.g. altruism, fairness, income, loyalty, price consciousness) and object-related (e.g. reference price, satisfaction) variables. Since the findings on the influence of social norm compliance are still inconclusive, further research is strongly needed. Future studies can build on the present approach to measure the effect of social norm compliance in laboratory experiments and benefit from the explanations of possible constraints that are offered in the present study. In addition, the findings offer implications to practitioners selling consumer goods under PWYW conditions or those consider doing so. For instance, sellers should take into account that anonymity in the purchasing process can lead to strong decrease of payments whereas a high degree of social interaction might lead to higher revenues.

\section{References}

[1] Kim, J.-Y., Natter, M. and Spann, M. (2009) Pay What You Want: A New Participative Pricing Mechanism. Journal of Marketing, 73, 44-58. http://dx.doi.org/10.1509/jmkg.73.1.44

[2] NME Blog (2012) Didradiohead’s “In Rainbows” Honesty Box Actually Damage the Music Industry? http://www.nme.com/blogs/nme-blogs/did-radioheads-in-rainbows-honesty-box-actually-damage-the-music-industry

[3] Gneezy, A., Gneezy, U., Riener, G. and Nelson, L.D. (2012) Pay-What-You-Want, Identity, and Self-Signaling in Markets. Proceedings of the National Academy of Sciences of the United States of America, 109, 7236-7240. http://dx.doi.org/10.1073/pnas.1120893109

[4] Fahmy, M. and Lee, V. (2009) Singapore Hotel Lets Guests Pay What They Want. Reuters. http://www.reuters.com/article/2009/02/04/us-singapore-hotel-idUSTRE5131MA20090204

[5] Uken, M. (2013) Zahle, so viel du willst! Zeit Online. http://www.zeit.de/wirtschaft/2013-01/pay-what-you-want

[6] Regner, T. and Riener, G. (2012) Voluntary Payments, Privacy and Social Pressure on the Internet: A Natural Field Experiment. DICE Discussion Paper: Vol. 82. DICE, Düsseldorf.

http://www.dice.hhu.de/fileadmin/redaktion/Fakultaeten/Wirtschaftswissenschaftliche Fakultaet/DICE/Discussion Pa per/082_Regner_Riener.pdf

[7] Bush, J. (2015) The Humble Visualisations! http://cheesetalks.net/humble/

[8] Riener, G. and Traxler, C. (2012) Norms, Moods, and Free Lunch: Longitudinal Evidence on Payments from a PayWhat-You-Want Restaurant. The Journal of Socio-Economics, 41, 476-483. http://dx.doi.org/10.1016/j.socec.2011.07.003

[9] Gneezy, A., Gneezy, U., Nelson, L.D. and Brown, A. (2010) Shared Social Responsibility: A Field Experiment in PayWhat-You-Want Pricing and Charitable Giving. Science, 329, 325-327. http://dx.doi.org/10.1126/science.1186744

[10] ElHarbi, S., Grolleau, G. and Bekir, I. (2014) Substituting Piracy with a Pay-What-You-Want Option: Does It Make Sense? European Journal of Law and Economics, 37, 277-297. http://dx.doi.org/10.1007/s10657-011-9287-y

[11] Kim, J.-Y., Kaufmann, K. and Stegemann, M. (2014) The Impact of Buyer-Seller Relationships and Reference Prices on the Effectiveness of the Pay What You Want Pricing Mechanism. Marketing Letters, 25, 409-423. http://dx.doi.org/10.1007/s11002-013-9261-2

[12] Kunter, M. (2015) Exploring the Pay-What-You-Want Payment Motivation. Journal of Business Research. http://dx.doi.org/10.1016/j.jbusres.2015.03.044

[13] Jang, H. and Chu, W. (2012) Are Consumers Acting Fairly toward Companies? An Examination of Pay-What-YouWant Pricing. Journal of Macromarketing, 32, 348-360. http://dx.doi.org/10.1177/0276146712448193

[14] Marett, K., Pearson, R. and Moore, R.S. (2012) Pay What You Want: An Exploratory Study of Social Exchange and Buyer-Determined Prices of iProducts. Communications of the Association for Information Systems, 30. http://aisel.aisnet.org/cais/vol30/iss1/10/

[15] Regner, T. (2014) Why Consumers Pay Voluntarily: Evidence from Online Music. Journal of Behavioral and Experimental Economics. 
[16] Jung, M.H., Nelson, L.D., Gneezy, A. and Gneezy, U. (2014) Paying More When Paying for Others. Journal of Personality and Social Psychology, 107, 414-431. http://dx.doi.org/10.1037/a0037345

[17] Mak, V., Zwick, R., Rao, A.R. and Pattaratanakun, J.A. (2015) “Pay What You Want” as Threshold Public Good Provision. Organizational Behavior and Human Decision Processes, 127, 30-43. http://dx.doi.org/10.1016/j.obhdp.2014.11.004

[18] Dorn, T. and Suessmair, A. (In Press) Is It Really Worth It? A Test of Pay-What-You-Want Pricing Strategies in a German Consumer Behavior Context. Global Business and Economics Review.

[19] Azar, O.H. (2003) The Social Norm of Tipping: A Review. SSRN Electronic Journal, 37, 380-402. http://dx.doi.org/10.2139/ssrn.370081

[20] Biel, A. and Thøgersen, J. (2007) Activation of Social Norms in Social Dilemmas: A Review of the Evidence and Reflections on the Implications for Environmental Behaviour. Journal of Economic Psychology, 28, 93-112. http://dx.doi.org/10.1016/j.joep.2006.03.003

[21] Bohnet, I. and Frey, B.S. (1999) Social Distance and Other-Regarding Behavior in Dictator Games: Comment. American Economic Review, 89, 335-339. http://dx.doi.org/10.1257/aer.89.1.335

[22] Charness, G. and Gneezy, U. (2008) What's in a Name? Anonymity and Social Distance in Dictator and Ultimatum Games. Journal of Economic Behavior \& Organization, 68, 29-35. http://dx.doi.org/10.1016/j.jebo.2008.03.001

[23] Hoffman, E., McCabe, K. and Smith, V.L. (1996) Social Distance and Other-Regarding Behaviour in Dictator Games. American Economic Review, 86, 653-660. http://www.jstor.org/stable/2118218

[24] Andreoni, J. and Petrie, R. (2004) Public Goods Experiments without Confidentiality: A Glimpse into Fund-Raising. Journal of Public Economics, 88, 1605-1623. http://dx.doi.org/10.1016/S0047-2727(03)00040-9

[25] Rege, M. and Telle, K. (2004) The Impact of Social Approval and Framing on Cooperation in Public Good Situations. Journal of Public Economics, 88, 1625-1644. http://dx.doi.org/10.1016/S0047-2727(03)00021-5

[26] Alpizar, F., Carlsson, F. and Johansson-Stenman, O. (2008) Anonymity, Reciprocity, and Conformity: Evidence from Voluntary Contributions to a National Park in Costa Rica. Journal of Public Economics, 92, 1047-1060. http://dx.doi.org/10.1016/j.jpubeco.2007.11.004

[27] List, J.A., Berrens, R.P., Bohara, A.K. and Kerkvliet, J. (2004) Examining the Role of Social Isolation on Stated Preferences. American Economic Review, 94, 741-752. http://dx.doi.org/10.1257/0002828041464614

[28] Nunes, J.C. and Boatwright, P. (2004) Incidental Prices and Their Effect on Willingness to Pay. Journal of Marketing Research, 41, 457-466. http://dx.doi.org/10.1509/jmkr.41.4.457.47014

[29] Chang, J.B., Lusk, J.L. and Norwood, F.B. (2009) How Closely Do Hypothetical Surveys and Laboratory Experiments Predict Field Behavior? American Journal of Agricultural Economics, 91, 518-534. http://dx.doi.org/10.1111/j.1467-8276.2008.01242.x

[30] Dunning, D., Heath, C. and Suls, J.M. (2004) Flawed Self-Assessment: Implications for Health, Education, and the Workplace. Psychological Science in the Public Interest, 5, 69-106. http://dx.doi.org/10.1111/j.1529-1006.2004.00018.x 